\title{
Evaluación de las Fuentes de Aguas Subterráneas y la Situación Actual de su Almacenamiento y Calidad en el Distrito de Vegueta 2012 - 2013
}

\author{
Evaluation of groundwater sources and the current situation of storage and quality \\ in the district of Vegueta 2012 - 2013
}

\author{
José Antonio Legua Cárdenas, Edwin Guillermo Gálvez Torres ${ }^{1}$, Ronald Luis Ramos Pacheco, \\ RESUMEN \\ Yasmin Vélez Chang ${ }^{1}$, Fredesvindo Fernández Herrera ${ }^{2}$
}

\begin{abstract}
Objetivo: Evaluar la calidad físico química y microbiológica del agua en base al servicio de análisis de laboratorio certificados(CERPER, SAG), así también de análisis in situ y de laboratorio de la Facultad de Ingeniería Química y Metalurgica. Materiales y Métodos: Los pozos de agua subterránea que están bajo el control por la asociaciones comunitarias en las zonas rurales presentaron mayores niveles de contaminación microbiológica moderamente altas como es el caso de contaminación por coliformes fecales y coliformes totales, salvo algunas excepciones, y este no es el caso de aquellos pozos que están bajo el control de EMAPA, que están también contaminados pero en niveles más bajos, en el caso de algunas zonas del distrito de Vegueta. Se encontró contaminación microbiológica en coliformes fecales y totales tanto en los pozos controlados por EMAPA en niveles bajos, en el caso de los pozos controlados por la población el nivel de contaminación fue mayor , a excepción del pozo de 200 millas, esta contaminación probablemente se debe al descuido al manipular los recipientes que se utilizan para extraer el agua o si es por bombeo las tapas de los pozos estan deterioradas y el pozo no esta en un ambiente cuidado y protegido. Algunos pozos de agua subterránea rebasan la concentración de nitratos recomendada por la Norma Técnica Peruana (NTP) para agua potable de consumo humano, que en ese caso es $45 \mathrm{mg} / \mathrm{L}$, encontrándose mayores valores al recomendado, probablemente esta contaminación es por la lixiviación de fertilizantes y materia orgánica que se mineraliza y el líquido lixiviante fluye hacia el acuífero contaminándolo, siente ese efecto mayor en aquellas zonas que tienen una menor altitud por la menor posibilidad de retención y absorción que tienen las sustancias nitrogenadas mineralizadas o en tránsito hacia la mineralización que fluyen hacia el acuífero, contaminándolo, poniendo en riesgo su calidad como agua potable. Resultados: Al respecto a la contaminación por metales pesado no se encontró ningún caso de la contaminación en los pozos evaluados tanto lo controlados por EMAPA, y también por aquellos controlados por las comunidades rurales. Conclusiones: La concentración en sulfatos, cloruro y nitratos estuvo moderamente altos por se está un característica muy propia de las aguas subterráneas que tienen presencia importante de estas sales y otras, respecto a las aguas superficiales que no tienen esta característica. Respecto a la capacidad del acuífero se reflejó como optima en los pozos de agua subterránea evaluados donde la diferencia entre el nivel de agua del pozo en reposo y el nivel de equilibrio cuando se bombeaba el agua de pozo hacia el exterior, una diferencia de aproximadamente $90 \mathrm{~cm}$ en los pozos evaluados.
\end{abstract}

Palabras clave: Evaluación, fuentes de agua, capacidad y calidad.

\footnotetext{
${ }^{1}$ Docente. Facultad de Ingeniería Química y Metalúrgica. Universidad Nacional José Faustino Sánchez Carrión. Email: jose.leguacardenas@ gmail.com

${ }^{2}$ Docente. Facultad de Ingenieria Agraria, Industrias Alimentarias y Ambiental, Universidad Nacional José Faustino Sánchez Carrión.
} 


\begin{abstract}
Objective: To evaluate the physical and microbiological quality of the water based on the certified laboratory analysis service (CERPER, SAG), as well as on-site and laboratory analysis of the Faculty of Chemical and Metallurgical Engineering. Materials and Methods Groundwater wells that are under the control of community associations in rural areas had higher levels of moderately high microbiological contamination such as fecal coliform and total coliform contamination, with few exceptions, and this did not ls the case of those wells that are under the control of EMAPA, which are also contaminated but at lower levels, in the case of some areas of the district of Vegueta. Microbiological contamination was found in fecal and total coliforms both in wells controlled by EMAPA at low levels, in the case of wells controlled by the population the level of contamination was higher, with the exception of the 200-mile well, this contamination is probably due By neglecting to manipulate the containers that are used to extract the water or if it is by pumping the lids of the wells are deteriorated and the well is not in a protected and protected environment. Some groundwater wells exceed the nitrate concentration recommended by the Peruvian Technical Standard (NTP) for drinking water for human consumption, which in this case is $45 \mathrm{mg} /$ $\mathrm{L}$, being higher values than recommended, probably this contamination is due to the leaching of Fertilizers and organic matter that mineralizes and the leaching liquid flows to the aquifer contaminating it, it feels that greater effect in those areas that have a lower altitude because of the less possibility of retention and absorption that the mineralized nitrogenous substances have or in transit towards the mineralization that Flow to the aquifer, contaminating it, putting its quality as a drinking water. Results: Regarding heavy metal contamination, no case of contamination was found in the wells evaluated, both controlled by EMAPA, and also by those controlled by rural communities. Conclusions: The concentration of sulphates, chloride and nitrates was moderately high because it is a very characteristic characteristic of groundwater that has an important presence of these salts and others, with respect to surface waters that do not have this characteristic. Regarding the aquifer capacity was reflected as optimal in the evaluated groundwater wells where the difference between the water level of the well at rest and the level of equilibrium when the well water was pumped out, a difference of approximately $90 \mathrm{Cm}$ in the wells evaluated.
\end{abstract}

Key words: Evaluation, water sources, capacity and quality.

\section{INTRODUCCIÓN}

El agua subterránea es una pequeña parte y a la vez gravitante de la masa de agua presente en los continentes, y se encuentra en los acuíferos bajo la superficie de la Tierra. El volumen del agua subterránea es mucho más importante que la masa de agua retenida en lagos o circulante, y aunque menor al de los mayores glaciares, las masas más extensas pueden alcanzar millones de kilómetros cuadrados. El agua del subsuelo es un recurso importante y de este se abastece a una tercera parte de la población mundial, pero de difícil gestión, por su sensibilidad a la contaminación y a la sobreexplotación. En el caso de distrito de Vegueta y los pozos de sus diferentes zonas rurales la contaminación se da por la aplicación indiscriminada y no técnica de plaguicidas y fertilizantes y por el descontrol en el manejo y cuidado de los pozos que están bajo el control de la comunidad y no de EMAPA.

El agua subterránea es parte de la precipitación que se filtra a través del suelo hasta llegar al material rocoso que está saturado de agua. El agua subterránea se mueve lentamente hacia los niveles bajos, generalmente en ángulos inclinados (debido a la gravedad) y eventualmente llegan a los arroyos, los lagos y al mar.

Es una creencia común que el agua subterránea llena cavidades y circula por galerías. Sin embargo, no siempre es así, pues puede encontrarse ocupando los intersticios (poros y grietas) del suelo, del sustrato rocoso o del sedimento sin consolidar, los cuales la contienen como una esponja. La única excepción significativa la ofrecen las rocas solubles, como las calizas y los yesos, susceptibles de sufrir el proceso llamado karstificación, en el que el agua excava simas, cavernas y otras vías de circulación.

\section{MATERIAL Y METODOS}

\section{Descripción del Área de Estudio}

El estudio se ha aplicado a los pozos de agua subterránea del distrito de Vegueta.

\section{Población y Muestra.}

La población o universo de la Investigación está constituido por el área de influencia del Valle de Huaura, perteneciente a la cuenca del río Huaura, que corresponde a las fuentes de aguas subterráneas (Pozos, Manantiales y Galería Filtrantes).

La muestra de la población está representada por las muestras de agua de los pozos representativos de Vegueta tanto de los pozos controlados por EMAPA y los pozos controlados por sectores de la comunidad. El muestreo se realizó siguiendo el protocolo respectivo tanto para los análisis para servicio de análisis de laboratorio certificado como también para los análisis realizados in situ y también en laboratorio de la Facultad de Ingeniería Química y Metalúrgica (FIQyM) de la UNJFSC.Población

La población para la presente investigación estará conformada por los desechos generados por el mercado municipal central de Huacho.

Materiales

Para el desarrollo del siguiente trabajo de investigación se emplearon los siguientes materiales: Muestras de agua de los pozos, galerías filtrantes de diferentes zonas del distrito de Vegueta. Equipo multiparàmetro, muestreador de agua, Kit para 
análisis de agua, balanzas digitales, vasos Erlenmeyer, medidor de nivel y profundidad de agua de pozo, Cooler para transporte de muestras de agua, linterna, guincha.

Soporte Informático: disco duro, USB, CD

Cámara fotográfica, se usó para captar la información en el momento oportuno, pues de esta manera se tendrá una presentación fidedigna.

Libreta de notas: Se anotó toda la información, características, puntos importantes u otros que sé consideró conveniente.

Método

Este es un caso de Investigación aplicada y un nivel de investigación explicativa.

El método de Investigación utilizado es el científico.

Diseño de Investigación: Diseño No Experimental, tipo transacional explicativo-causal.

El procedimiento de esta investigación se inició con la coordinación con las autoridades municipalidad distrital de Vegueta, específicamente con su área ambiental, respecto a los principales pozos e control así también con los pozos nuevos y con aquellos que ya no utilizan o están fuera de control. Así también se coordinó con las representantes de la Autoridad Nacional del Agua(ANA) y de EMAPA.

Se recolectó información de la calidad físico química y microbiológica del agua en base de servicio de análisis de laboratorio certificados(CERPER, SAG), así también de análisis in situ y de laboratorio de la FIQyM, en este caso de recolección de datos también se recolectó información del nivel de recarga con equipos propios del proyecto.

El procesamiento de la información se realizó de manera computarizada; con el fin de obtener resultados para contrastar con los objetivos e hipótesis planteadas en la investigación; utilizando el Análisis de Varianza del Excel.

El ámbito de estudio es local, comprendió aquellos pozos de aguas subterráneas de zonas de mayor densidad demográfica y mayor consumo de agua tales como: Primavera, Mazo, Primavera, La laguna, Santa Fé, 200 millas, San Isidro y La Perlita.

\section{RESULTADOS}

Los resultados se presentan en el Tabla 1, Tabla 2 y Tabla 3 donde se presentan resultados de análisis físico químicos y microbiológico, para muestras de agua tomadas según protocolo de pozos de agua subterránea de las zonas de: Primavera, La laguna, Mazo, 200 millas, Santa Fe, San Luis y Galerías Filtrantes.

\section{DISCUSIÓN}

Los resultados en general presentan una tendencia respecto a su calidad físico química y microbiológica a no cumplir con las normas de calidad, aquellos pozos que están bajo el control de las comunidades, en el caso de los pozos que están bajo el control de EMAPA, se presentan casos de contaminación microbiológica pero no tan importante como se presenta en el caso de los pozos que son administrados por las comunidades rurales, a excepción del pozo de agua subterránea de 200 millas.

En relación a la contaminación por nitratos se observa que a medida que se disminuye la altitud se incrementa la concentración en nitratos, rebasando el la concentración máxima recomendada por la norma técnica peruana(NTP).

Respecto a la contaminación por coliformes fecales esta esta es más excesiva en la galerías filtrantes cercanas a la La Laguna, las cuales se mezclan con aguas de regadío contaminadas y también en el pozo de agua subterránea de la zona de San Luis se presentó contaminación por coliformes fecales, así también es mayor la contaminación por coliformes fecales en el caso de aquellos pozos que están bajo la administración de la comunidad rural, a excepción del pozo de la zona de 200 millas, tal como se ilustra en las Tablas.

En el caso de la contaminación por coliformes totales se refleja la misma tendencia y explicación que se presentó para la contaminación por coliformes fecales, tal como se ilustra en la Tabla 3.

En cuanto a contaminación por metales pesados no se presentó ningún caso de contaminación que exceda lo recomendado por la NTP, así también en el caso de los otros metales tampoco se verifico contaminación.

El nivel de recarga de casi todos los pasos evaluados es óptimo, en razón a cuando se midió al altura dinámica de los pozos fluctuó alrededor de $90 \mathrm{~cm}$ dependiendo de la profundidad y batimetría del lugar donde se ubica el pozo, esta medición se hizo cuando estando estable el nivel del pozo se bombeo el agua de pozo para cargar los tanques de almacenamiento, bajaba el nivel mínimo en el pozo que pese a seguir bombeando agua se mantenía constante el nivel de agua en el pozo y cuando se dejó de bombear se recargaba el pozo por la presión dinámica de las aguas subterráneas del acuífero, este nivel diferencial que se registro fue de alrededor de $90 \mathrm{~cm}$ en la mayoría de los pozos evaluados en el distrito de Vegueta.

También se utilizó el kit para análisis de muestras de agua y el equipo multiparametro de laboratorio adquiridos en el proyecto para complementar la evaluación fisicoquímica realizada por servicios de laboratorio a empresas particulares, a fin de complementar y corroborar los resultados encontrado que se muestran en la Tabla 1, Tabla 2.

Así por ejemplo se muestreo aguas de pozos en octubre del 2014 en las zonas de Vegueta, encontrando los siguientes resultados: 
Tabla 1: Evaluación Físico Química de zonas de Vegueta-Octubre.

\begin{tabular}{|c|c|c|c|c|}
\hline \multicolumn{5}{|c|}{ Zonas de Vegueta } \\
\hline $\begin{array}{l}\text { Parámetro } \\
\text { Fisico- } \\
\text { Quimico }\end{array}$ & Primavera & La Laguna & La Perlita & $\begin{array}{l}\text { Galerías } \\
\text { Filtrantes } \\
\text { de la } \\
\text { Laguna }\end{array}$ \\
\hline $\begin{array}{l}\text { Nitrato } \\
(p p m)\end{array}$ & 6 & - & 10 & 4 \\
\hline $\begin{array}{l}\text { Alcalinidad } \\
\text { (ppm) }\end{array}$ & 148 & 143 & 268 & 380 \\
\hline $\begin{array}{l}\text { Potasio } \\
\text { (ppm) }\end{array}$ & 488 & - & 483 & - \\
\hline $\mathrm{Ph}$ & 7.42 & 7.43 & 7.60 & 7.9 \\
\hline $\begin{array}{l}\text { Resistividad } \\
\text { (Ohm) }\end{array}$ & 482 & 495 & 489 & 499 \\
\hline $\begin{array}{l}\text { Conductividad } \\
(\mathrm{mS} / \mathrm{cm})\end{array}$ & 2,068 & 1.992 & 2.06 & 2.025 \\
\hline Salinidad (ppt) & 0.88 & 0.81 & 0.82 & 0.89 \\
\hline TDS (ppt) & 1.23 & 1.23 & 1.27 & 1.29 \\
\hline Temperatura $\left({ }^{\circ} \mathrm{C}\right)$ & C) 26 & 26 & 26 & 22 \\
\hline
\end{tabular}

Fuente: Propia

Tabla 2: Evaluación Físico Química de zonas de Vegueta - Noviembre.

\begin{tabular}{|c|c|c|c|c|c|}
\hline \multicolumn{6}{|c|}{ Zonas de Vegueta } \\
\hline $\begin{array}{l}\text { Parámetro } \\
\text { Fisico- } \\
\text { Quimico }\end{array}$ & Primavera & $\begin{array}{c}\text { La } \\
\text { Laguna }\end{array}$ & $\begin{array}{c}\text { La } \\
\text { Perlita }\end{array}$ & Mazo & $\begin{array}{c}200 \\
\text { millas }\end{array}$ \\
\hline $\begin{array}{l}\text { Nitrato } \\
\text { (ppm) }\end{array}$ & 7 & 8 & 10 & 4 & 7 \\
\hline $\begin{array}{l}\text { Alcalinidad } \\
\text { (ppm) }\end{array}$ & 145 & 170 & 260 & 385 & 185 \\
\hline $\begin{array}{l}\text { Potasio } \\
\text { (ppm) }\end{array}$ & 480 & 490 & 481 & 422 & 423 \\
\hline $\mathrm{Ph}$ & 7.42 & 7.43 & 7.60 & 7.9 & 7.5 \\
\hline $\begin{array}{l}\text { Resistividad } \\
\text { (Ohm) }\end{array}$ & 482 & 495 & 489 & 499 & 490 \\
\hline $\begin{array}{l}\text { Conductividad } \\
(\mathrm{mS} / \mathrm{cm})\end{array}$ & 2,025 & 1.973 & 2.05 & 2.0 & 2.04 \\
\hline Salinidad (ppt) & 0.88 & 0.81 & 0.82 & 0.89 & 0.84 \\
\hline TDS (ppt) & 1.22 & 1.223 & 1.26 & 1.28 & 1.24 \\
\hline Temperatura $\left({ }^{\circ} \mathrm{C}\right)$ & C) 26 & 26 & 26 & 23 & 26 \\
\hline
\end{tabular}

Fuente: Propia
Tabla 3: Evaluación Microbiológica de zonas de Vegueta

\begin{tabular}{lccccc}
\hline \multicolumn{5}{c}{ Zonas de Vegueta } \\
\hline $\begin{array}{l}\text { Parámetro } \\
\text { Microbiológ } \\
\text { ico }\end{array}$ & Primavera & $\begin{array}{c}\text { San } \\
\text { Luis }\end{array}$ & $\begin{array}{c}\text { Santa } \\
\text { Fe }\end{array}$ & Mazo & $\begin{array}{c}\mathbf{2 0 0} \\
\text { millas }\end{array}$ \\
\hline $\begin{array}{l}\text { Coliformes } \\
\text { Fecales }\end{array}$ & $<1.8$ & 17 & 11 & $<1.8$ & $<1.8$ \\
$\begin{array}{l}\text { Coliformes } \\
\text { Termotolerantes }\end{array}$ & & & & 200 & \\
$\begin{array}{l}\text { Coliformes } \\
\text { Totales }\end{array}$ & 49 & 28000 & 79 & 4200 & $<1.8$ \\
\hline \begin{tabular}{l} 
Fuente: Propia \\
\hline
\end{tabular} & & & & & \\
\hline
\end{tabular}

\section{CONCLUSIONES}

Primera: Los pozos de agua subterránea que están bajo el control por la asociaciones comunitarias en las zonas rurales presentaron mayores niveles de contaminación microbiológica moderadamente altos como es el caso de contaminación por coliformes fecales y coliformes totales, salvo algunas excepciones, y este no es el caso de aquellos pozos que están bajo el control del EMAPA, que están también contaminados pero en niveles mas bajos, en el caso de algunas zonas del distrito de Vegueta.

Segunda: Se encontró contaminación microbiológica en coliformes fecales y totales tan to en los pozos controlados por EMAPA en niveles bajos salvo el caso de la zona de La Laguna que registro un alto nivel de contaminación en coliformes totales, en el caso de los pozos controlados por la población el nivel de contaminación fue mayor , a excepción del pozo de 200 millas, esta contaminación probablemente se debe al descuido al manipular los recipientes que se utilizan para extraer el agua o si es por bombeo las tapas de los pozos no son eficientes o no lo tienen.

Tercera: Algunos pozos de agua subterránea rebasan la concentración de nitratos recomendada por la Norma Técnica Peruana (NTP) para agua potable de consumo humano, que en ese caso es $45 \mathrm{mg} / \mathrm{L}$, encontrándose mayores valores al recomendado, probablemente esta contaminación es por la lixiviación de fertilizantes y materia orgánica que se mineraliza y el líquido lixiviante fluye hacia el acuífero contaminándolo, siente ese efecto mayor en aquellas zonas que tienen una menor altitud por la menor posibilidad de retención y absorción que tienen las sustancias nitrogenadas mineralizadas o en tránsito hacia la mineralización que fluyen hacia el acuífero, contaminándolo, poniendo en riesgo su calidad como agua potable.

Cuarta: Por otro lado respecto a la contaminación por metales pesado no se encontró ningún caso en los pozos evaluados tanto lo controlados por EMAPA, y también por aquellos controlados por las comunidades rurales. 
Cinco: La concentración en sulfatos, cloruro y nitratos estuvo moderadamente altos por se está un característica muy propia de las aguas subterráneas que tienen presencia importante de estas sales y otras, respecto a las aguas superficiales que no tienen esta característica.

Sexto: Respecto a la capacidad del acuífero se reflejó como optima en los pozos de agua subterránea evaluados donde la diferencia entre el nivel de agua del pozo en reposo y el nivel de equilibrio cuando se bombeaba el agua de pozo hacia el exterior, una diferencia de aproximadamente $90 \mathrm{~cm}$ en los pozos evaluados

\section{REFERENCIAS BIBLIOGRÁFICAS}

Ben Aakame, R.(2015). Evaluación de la calidad físico-química del agua de las aguas subterráneas en las zonas del noroeste de Marruecos y del peligro de la Salud. En Diario de Materiales y Ciencias Ambientales. Volumen 6, Número 5, 2015, páginas 1228-123. Editorial: Mohammed Premier Universidad. Rabat Marruecos.

Dahiya, S.A.(2007). Análisis de la calidad del agua subterránea mediante la evaluación sintética difusa. En DKushwah Journal de Materiales Peligrosos. Volumen 147, Número 3, páginas 938-946.

Pizzol L. (2015). Metodología de priorización basada en el riesgo para la clasificación de las fuentes de contaminación de las aguas subterráneas. En Ciencia of the Total Environment. Volumen 506507,págs. 505-517. Departamento de Ciencias
Ambientales, Informática y Estadística, Universidad Ca'Foscari de Venecia, Italia.

Stathatou P. at el. (2015). Vulnerabilidad de los sistemas de agua: un marco global para su evaluación e identificación de estrategias de adaptación (Publicación en Prensa). Taylor and Francis Inc.

Tziritis E. at el. (2014) Desarrollo de un índice operacional de la calidad del agua (punto de venta) como una herramienta versátil para ayudar a la gestión de los recursos hídricos subterráneos y la planificación estratégic. En Diario de Hidrología, Volumen 517, páginas 339350. Organización Agrícola Helénica, Land Institute Reclamación, Sindos, Grecia. 